\title{
Cognitive predictors for five-year conversion to dementia in community-dwelling Chinese older adults
}

\author{
Candy H. Y. Wong, ${ }^{1}$ Grace T. Y. Leung, ${ }^{1}$ Ada W. T. Fung, ${ }^{2}$ W. C. Chan ${ }^{3}$ \\ and Linda C. W. Lam² \\ ${ }^{1}$ Department of Psychiatry, Tai Po Hospital, Tai Po, Hong Kong \\ ${ }^{2}$ Department of Psychiatry, The Chinese University of Hong Kong, Tai Po Hospital, Tai Po, Hong Kong \\ ${ }^{3}$ Department of Psychiatry, The University of Hong Kong, Queen Mary Hospital, Pokfulam, Hong Kong
}

Background: This study evaluates which cognitive measure is best for predicting incident dementia in a population-based random sample of Chinese older adults without dementia over a five-year period.

Methods: A total of 787 community-dwelling Chinese older adults without dementia in Hong Kong were assessed at baseline (T0), at two years (T1), and at five years (T2).

Results: The annual conversion rate to dementia was $1.6 \%$ and $6.3 \%$ for baseline normal participants and baseline mild cognitive impairment (MCI) participants, respectively. The Cantonese version of Mini-mental State Examination (CMMSE) scores declined significantly over time. In participants who progressed to dementia, Category Verbal Fluency Test (CVFT) scores dropped significantly from T0 to T1. A 1-SD drop of either CMMSE or CVFT in two years predicted dementia at five years with $91.5 \%$ sensitivity and $62.0 \%$ specificity. A stable CMMSE and CVFT at two years predicted a $91 \%$ chance of not progressing to clinical dementia at five years.

Conclusion: In this community sample of Chinese older adults, a decline in cognitive screening tests in short term (two years) offered useful information in predicting dementia conversion over a longer period.

Key words: cognitive predictors, dementia, mild cognitive impairment

\section{Background}

Dementia brings a significant burden to the healthcare system worldwide. Clinical trials on possible interventions preventing or delaying the onset of dementia are on rapid development. The greatest challenge for successful prevention is early identification of cognitively at-risk individuals. The use of cognitive tests as a predictor for decline in the community is of special relevance in regions where neuroimaging and cerebrospinal fluid (CSF) biomarkers are not widely available.

Decline in cognitive function began years before the clinical diagnosis of dementia (Hall et al., 2000; Grober et al., 2008; Johnson et al., 2009). The trajectories of cognitive decline are highly variable among participants. Episodic memory is not the only cognitive domain being affected at

Correspondence should be addressed to: Dr Candy H. Y. Wong, Department of Psychiatry, Tai Po Hospital, 9 Chuen On Road, Tai Po, N. T., Hong Kong. Phone: +(852) 26076026. Email: whycandy@gmail.com. Received 14 Sep 2012; revision requested 17 Oct 2012; revised version received 17 Jan 2013; accepted 23 Jan 2013. First published online 2 April 2013. the preclinical phase. In the Baltimore Longitudinal Study of Aging (BLSA), it was shown that memory performance predicted future Alzheimer's dementia sensitively and early in the decline, while measures on executive dysfunction become more discriminating toward the time of diagnosis (Rubin et al., 1998). A sharp inflection point followed by accelerating decline exists in multiple cognitive domains in preclinical dementia (Johnson et al., 2009).

Cognitive decline may also occur in the absence of significant neurodegenerative pathologies (Ebly et al., 1995). Some studies reported selective effects on certain cognitive domains and some studies reported a global effect. Visual memory, semantic memory, and executive function were susceptible to age-related decline in BLSA (Grober et al., 2008). A global decline in all cognitive domains was reported with increasing age, including general cognitive ability, episodic memory, primary memory, verbal fluency, and visuospatial ability (Small et al., 2003). Significant decline was observed in global cognition, episodic memory, semantic memory, working 
memory, perceptual speed, but not visuospatial ability in another study (Wilson et al., 2003). It was also reported that cognitive performance remained stable in participants without dementia (Galvin et al., 2005). Early detection focusing on episodic memory tasks only may not be sensitive enough to detect subsequent dementia cases. Other cognitive domains have also been shown to have significant decline in the preclinical phase, like visuospatial ability (Johnson et al., 2009), verbal memory (Howieson et al., 1997), and executive function (Chen et al., 2001).

Data from local studies previously studied volunteers in the community with subjective memory complaints (Lam et al., 2008c). The study aimed at validating a short cognitive screening test for mild cognitive impairment (MCI) and mild dementia. The Chinese Abbreviated Mild Cognitive Impairment (CAMCI) Test consists of shortened form of the Category Verbal Fluency Test (CVFT) and delayed recall. It was shown that the battery was sensitive in detecting MCI in both phase 1 , comprising of volunteers from social centers and residential hostels, and phase 2, comprising of population-based random samples. A subsequent 20-month follow-up showed that the CAMCI was able to identify participants with higher likelihood of progression to dementia. The group being followed up was the volunteer participants from phase 1, with lower education level and was older. Besides, by responding to the advertisement of the study, this group of participants was more likely to have subjective memory complaints and the result might not be projectable to the whole population. Followup data from a population-based sample with a longer follow-up period are needed to derive suitable paradigm for screening purpose.

The objectives of this study were to examine the longitudinal cognitive profiles in Chinese older adults without dementia in Hong Kong. We hoped to identify early cognitive predictors of dementia in the community to capture early changes.

\section{Methods}

\section{Study design and participants}

This is a five-year follow-up study from a population-based community survey of the prevalence of cognitive impairment in Chinese older people in Hong Kong, the Hong Kong Memory and Ageing Prospective Study (HK-MAPS). The baseline survey was conducted from October 2005 to July 2006. In short, a total of 6,100 people aged 60 years or above were randomly sampled from a non-institutionalized population in Hong Kong and were screened using the Cantonese version of the Mini-mental State Examination (CMMSE) and the Abbreviated Memory Inventory for the Chinese (AMIC). Local validated cut-offs used for CMMSE were 18 and below, 20 and below, 22 and below for participants with no education, one to two years of education, and more than two years of education, respectively. The cutoff used for AMIC was three or above (Lam et al., 2008a). Those who were screen-positive and a randomly selected $5 \%$ of the screen-negative participants were invited for further assessment for dementia following a standardized protocol. Clinical dementia was defined according to the criteria of the Diagnostic and Statistical Manual of the Mental Disorders, 4th edition (DSM-IV, American Psychiatric Association, 2000). Nine hundred and thirty-one participants completed the assessment and 144 had a diagnosis of dementia. The remaining 787 participants constituted the sample for this study.

The participants were assessed at baseline (T0), at two years (T1), and at five years (T2). The baseline assessment was conducted from October 2005 to July 2006, the first follow-up from August 2007 to June 2008, and the second follow-up from March 2011 to February 2012. Participants who agreed to a follow-up assessment were contacted by phone and/or by post. Participants who skipped the first follow-up were also invited to take part in the second follow-up. Interviews were conducted at their place of residence or at a regional social/health center. The trained research assistants and psychiatrists collected information by face-toface assessments using standardized questionnaires and cognitive tests. Before commencement of the interview, written informed consent was obtained from each participant or from a first-degree relative if the participant was not able to give consent. The study was approved by the ethics committee of the Chinese University of Hong Kong and the Department of Health of the Government of the Hong Kong Special Administrative Region.

\section{Assessment}

\section{BACKGROUND PERSONAL AND CLINICAL}

\section{DATA}

Sociodemographic data and lifestyle characteristics were obtained. Self-rated health and mobility were recorded. Additional information was obtained from informants available and by inspecting prescribed medications and/or medical records kept by the participants. Self-reported medical history was recorded using the Cumulative Illness Rating Scale (CIRS) (Conwell et al., 1993). The CIRS gives a cumulative score as a measure of impairment due to physical illness in 13 organ systems. A 
higher score indicates a greater burden of physical illness. Depressive symptoms were assessed using the Cornell Scale for Depression in Dementia (CSDD; Alexopoulos et al., 1988).

\section{Measures of cognitive function}

Diagnoses of dementia were made by a trained psychiatrist with DSM-IV TR criteria. The severity of cognitive impairment was determined by the global Clinical Dementia Rating (CDR). CDR is a semi-structured clinical interview rated with a scoring from 0 to 3 along six dimensions including memory, orientation, judgment, community affairs, hobbies and habits, and personal care. The criteria of MCI follow a combined clinical and cognitive model used in a previous study, evaluated to be more practical in MCI screening in the local aged population (Lam et al., 2010). The criteria of amnesic MCI include (1) subjective complaints of memory loss or cognitive impairment; (2) objective memory impairment of delay learning scores being $\geq 1.5$ SD below education- and agematched persons with CDR 0; (3) relatively intact activities of daily living; and (4) clinically without dementia $(C D R<1)$. Non-amnesic $M C I$ will be identified with a memory score above the -1.5 SD cut-off, but non-memory cognitive scores below 1-SD cut-off, two or more non-memory domains in CDR scored $>0$. Both amnesic and non-amnesic MCI were included in this study.

Cognitive assessments included the Chinese version of the Alzheimer's Disease Assessment ScaleCognitive Subscale (ADAS-Cog), the CMMSE, ten-minute delayed recall, the CVFT, and the trail making tests ( $\mathrm{A}$ and $\mathrm{B}$ ).

1. The Chinese ADAS-Cog is an 11-item scale with scores ranging from 0 to 70 . Higher scores indicate greater severity. It includes components of memory, language, and praxis. It is considered a sensitive test to monitor changes in cognitive function (Rosen et al., 1984; Chu et al., 2000).

2. The CMMSE is a standard clinical test for global cognitive function (Folstein et al., 1975). The maximum score is 30 . It assesses orientation, registration, attention, recall, language, and visual construction. It has been validated locally (Chiu et al., 1994).

3. The ten-minute delayed recall was an extension of the first part of the ADAS-Cog. In ADAS-Cog, participants were required to recall the ten words after a learning trial with ten minutes delay.

4. The CVFT is a test of language, semantic memory, and executive function. Participants were asked to generate as many exemplars as possible from semantic categories including animals, fruits, and vegetables, in one minute for each category (Chiu et al., 1997).
5. The trail making test is a test providing information about visual search speed, scanning, speed of processing, mental flexibility, as well as executive functioning (Bowie and Harvey, 2006). There are two parts to the Chinese trail test: $\mathrm{A}$, in which the subject needs to connect numbers in sequential order, and $\mathrm{B}$, in which the subject alternates between Arabic and Chinese to suit the literacy of local older population.

\section{Statistical analyses}

The sample size estimation was performed using the Power and Precision software version 3.0 (Biostat, Englewood, New Jersey, USA). From the same group community-dwelling older adults without dementia, the two-year conversion rate was 5.3\% (Leung, 2008). For sample size estimates for computation of significant predictors, logistic regression with diagnosis of dementia by CDR as dependent variable was adopted. For 100 cases of dementia identified, the power for estimation of risk factors would be $80 \%$ power ( $\alpha$ of 0.05 ). Assuming that the deterioration rate is uniform, the fiveyear conversion rate would be $13.25 \%$ and about 104 participants are expected to develop dementia. The sample size available at the baseline should be able to achieve satisfactory power $(82 \%)$.

All other analyses were performed using the Statistical Package for Social Sciences (SPSS) software (Chicago, Illinois, USA). Baseline characteristics assuming normal distribution were compared by Student's $t$-test, non-parametric variables by the Mann-Whitney $U$ test, and categorical data by the $\chi^{2}$ test. The trajectory of changes over time for each specific cognitive domain was determined. In addition to studying the absolute change in scores, changes in cognitive domains were computed by $Z$ scores at specific time points with reference to the baseline performance of age- and educational level-matched participants with a CDR of 0 . Test scores at different time points were compared by a paired- $T$ test. Statistical significance was set at $\mathrm{p} \leq 0.05$. In a previous local study, MCI participants were shown to differ from normal participants by $1 \mathrm{SD}$ in cognitive scores (Lam et al., 2008b). Thus, a 1-SD drop was used in analysis in this study as a significant drop in cognitive performance. Multivariate logistic regression analyses were performed to examine possible baseline predictors of incident dementia.

\section{Results}

\section{Baseline characteristics}

In the 787 participants, $523(66.5 \%)$ were cognitively normal and $264(33.5 \%)$ were MCI 


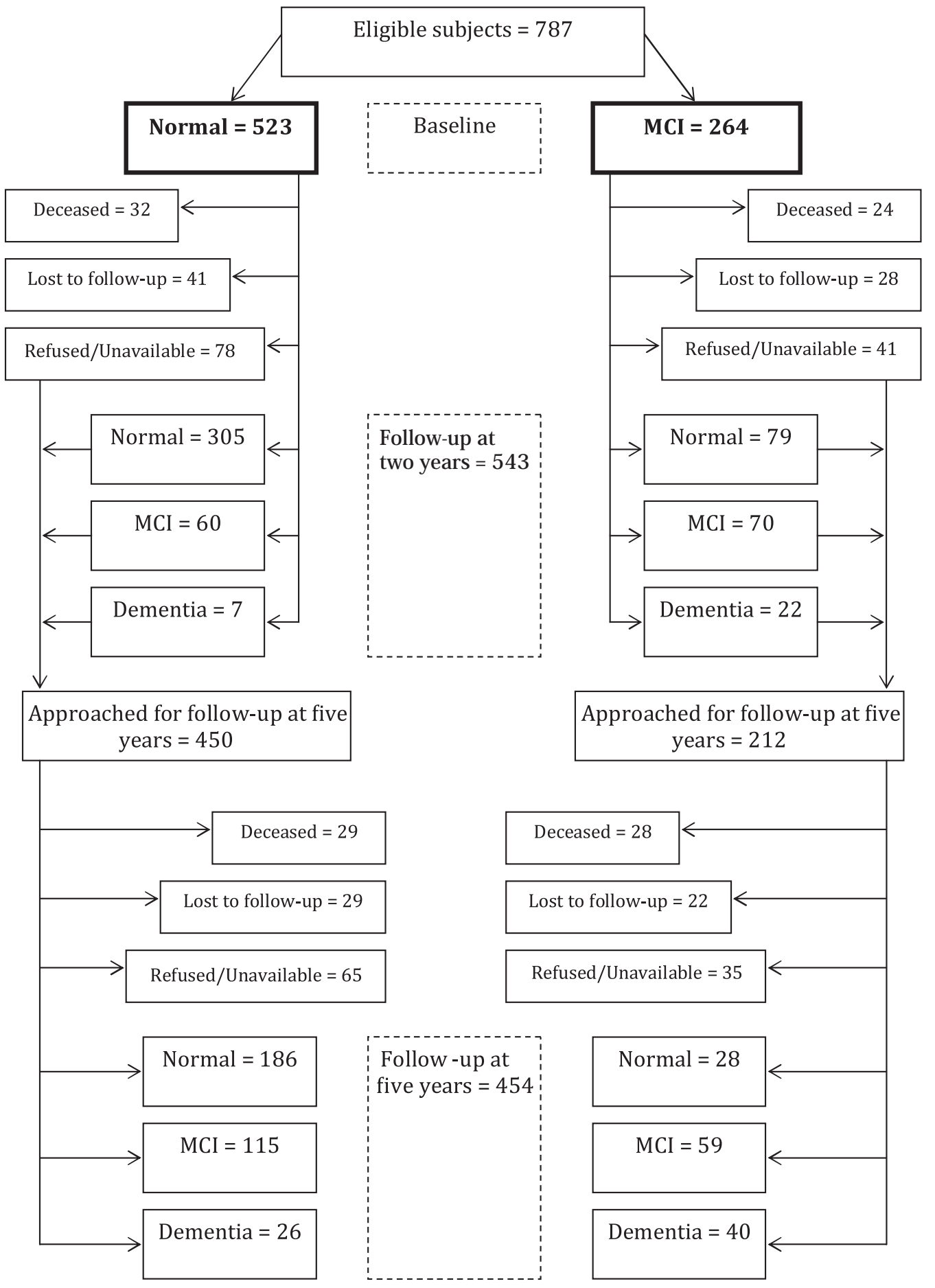

Figure 1. Flow chart of clinical outcome at five years.

at baseline (T0). A total of 543 participants participated in the first follow-up (T1). By five years (T2), $113(14.4 \%)$ died and $120(15.2 \%)$ were lost to follow-up. In the remaining 554 participants, 100 $(18.1 \%)$ declined participation or were not available for assessment (due to hospitalization or being away from Hong Kong). Therefore, 454 participants were assessed in the second follow-up (T2): 327 of them were normal at baseline and 127 were MCI at baseline (Figure 1). The mean follow-up interval of the first follow-up was 22.3 months, and that of the second follow-up was 64.7 months. The sample had a mean age of 77.0 years at five years $(\mathrm{SD}=6.7$, range $=65-100), 44.9 \%$ were male, mean education was 4.9 years $(\mathrm{SD}=4.7$, range $=0$ 20) and the baseline mean CMMSE score was 26.1 $(\mathrm{SD}=2.6$, range $=16-30) \quad($ Table 1$)$. The differences in baseline characteristics between those 
Table 1. Demographics characteristics for participants assessed at five years $(N=454)$

\begin{tabular}{lcc}
\hline & $\begin{array}{l}\text { MEAN/ } \\
\text { FREQUENCY }\end{array}$ & SD (RANGE)/\% \\
\cline { 1 - 2 }$\ldots \ldots \ldots \ldots \ldots \ldots$ \\
Age (years) & 77.0 & $6.7(65-100)$ \\
Sex & & \\
$\quad$ Male & 204 & $44.9 \%$ \\
$\quad$ Female & 250 & $55.1 \%$ \\
Education (years) & 4.9 & $4.7(0-20)$ \\
Baseline characteristics & & \\
CMMSE & 26.1 & $2.6(16-30)$ \\
CVFT total & 34.3 & 8.6 \\
Delayed recall & 5.4 & 2.6 \\
ADAS-Cog & 11.0 & 5.4 \\
CIRS total & 2.7 & 2.2 \\
CSDD total & 0.9 & 2.3 \\
\hline
\end{tabular}

CMMSE = Cantonese version of the Mini-mental State Examination; $\mathrm{CVFT}=$ Category Verbal Fluency Test; ADAS$\mathrm{Cog}=$ Chinese version of the Alzheimer's Disease Assessment Scale-Cognitive Subscale; CSDD = Cornell Scale for Depression in Dementia; CIRS = Cumulative Illness Rating Scale; $\mathrm{SD}=$ standard deviation.

who were assessed at five years and those who did not but were alive were evaluated (Table 2). The participants who were assessed at T2 had better cognitive function at baseline with higher CMMSE $(p=0.031)$, CVFT $(p=0.001)$, delayed recall score $(p=0.005)$, and lower ADAS-Cog total score $(p=0.007)$. They had higher education level $(p=0.013)$. There was no difference in age and gender.

\section{Progression to dementia at five years}

Table 3 shows the follow-up status of participants at the second follow-up. For the baseline normal participants, $56.9 \%$ remained cognitively normal; $46.5 \%$ of the MCI participants stayed as MCI. The five-year conversion rate into dementia was $7.9 \%$ and $31.5 \%$ for baseline normal and MCI participants, respectively $\left(\chi^{2}=40.8, \mathrm{p}<0.001\right)$. The participants who progressed were older $(p<0.001)$, less educated $(p<0.001)$, and had lower cognitive scores at baseline (CMMSE, CVFT, delayed recall, and Trail A, $p<0.001$; Trail $\mathrm{B}, \mathrm{p}=0.001)$.

\section{Change in cognitive profile across the five years}

The drop in CMMSE scores was significant across the five years $(p<0.001)$. The annual drop in CMMSE scores from $\mathrm{T} 1$ to $\mathrm{T} 2$ was more than from $\mathrm{T} 0$ to $\mathrm{T} 1$, indicating a more rapid decline with time (Figure 2). An early drop in CVFT was found in participants who progressed to clinical dementia at $\mathrm{T} 2(\mathrm{p}=0.388)$ (Figure 3). The drop in ten-minute delayed recall and Trail $\mathrm{B}$ was also significant ( $p<0.001, p=0.009$, respectively). The changes in ADAS-Cog and Trail A were not significant.

\section{Baseline predictors of subsequent progression to dementia at five years}

Baseline characteristics differed significantly between the participants who remained stable and those who developed dementia at five years (age, years of education, CMMSE, CVFT, delayed recall, and ADAS-Cog $\mathrm{p}<0.001$; CSDD $p=0.001 ;$ CIRS $p=0.016$; sex: $\chi^{2}=15.39$, $\mathrm{p}<0.001)$. These baseline characteristics were put into logistic regression model and results were shown in Table 4. CMMSE and delayed recall scores at baseline were shown to be significant

Table 2. Comparison of the baseline characteristics of participants who were assessed at second follow-up with those who did not

\begin{tabular}{|c|c|c|c|}
\hline $\begin{array}{l}\text { CHARACTERISTICS } \\
\text { AT BASELINE }\end{array}$ & $\begin{array}{l}\text { PARTICIPANTS } \\
\text { ASSESSED AT } 2 \text { nd } \\
\text { FU }(S D)(N=454)\end{array}$ & $\begin{array}{l}\text { ALIVE } \\
\text { PARTICIPANTS } \\
\text { MISSING AT 2nd FU } \\
(\mathrm{SD})(\mathrm{N}=220)\end{array}$ & $P$ \\
\hline CMMSE & $26.1(2.63)$ & $25.6(2.68)$ & $0.031^{*}$ \\
\hline CVFT total & $34.3(8.58)$ & $32.0(8.07)$ & $0.001^{*}$ \\
\hline Delayed recall & $5.4(2.61)$ & $4.5(2.79)$ & $0.005^{*}$ \\
\hline ADAS-Cog & $11.0(5.40)$ & $12.7(5.49)$ & $0.007^{*}$ \\
\hline Age & $71.4(6.73)$ & $72.0(7.17)$ & 0.346 \\
\hline Education (years)\# & $5.1(4.76)$ & $4.2(4.48)$ & $0.013^{*}$ \\
\hline $\operatorname{Sex}(M: F)$ & $204: 250$ & $91: 129$ & 0.381 \\
\hline
\end{tabular}

Note. p value of sex by $\chi^{2}$; \# by Mann-Whitney $U$ test; others by $t$-test.

${ }^{*} \mathrm{p}=\leq 0.05$.

CMMSE $=$ Cantonese version of the Mini-mental State Examination; CVFT $=$ Category Verbal

Fluency Test; ADAS-Cog = Chinese version of the Alzheimer's Disease Assessment Scale-Cognitive Subscale; 2nd FU = second follow-up. 
Table 3. Cognitive status at five years $(N=787)$

\begin{tabular}{|c|c|c|c|c|c|}
\hline \multirow[b]{2}{*}{$\begin{array}{l}\text { BASELINE COGNITIVE } \\
\text { STATUS }\end{array}$} & \multicolumn{5}{|c|}{ COGNITIVE STATUS AFTER FIVE YEARS } \\
\hline & NORMAL & MCI & $\begin{array}{l}\text { MILD } \\
\text { DEMENTIA }\end{array}$ & $\begin{array}{l}\text { MODERATE } \\
\text { DEMENTIA }\end{array}$ & $\begin{array}{l}\text { SEVERE } \\
\text { DEMENTIA }\end{array}$ \\
\hline Normal $(N=523)$ & $186(56.9 \%)$ & $115(35.2 \%)$ & $20(6.1 \%)$ & $6(1.8 \%)$ & 0 \\
\hline $\operatorname{MCI}(N=264)$ & $28(22 \%)$ & $59(46.5 \%)$ & $25(19.7 \%)$ & $13(10.2 \%)$ & $2(1.6 \%)$ \\
\hline
\end{tabular}

$\mathrm{MCI}=$ mild cognitive impairment.

Table 4. Baseline predictors of subsequent dementia by logistic regression

\begin{tabular}{lrllll}
\hline & \multicolumn{1}{c}{ B } & SE & WALD & p & EXP (B) (95\% CI $)$ \\
Age & 0.036 & 0.055 & 0.445 & 0.505 & $1.037(0.932-1.154)$ \\
Edu yrs & -0.071 & 0.112 & 0.401 & 0.526 & $0.932(0.748-1.160)$ \\
Sex & 0.115 & 0.700 & 0.027 & 0.870 & $1.121(0.284-4.426)$ \\
CMMSE & -0.312 & 0.130 & 5.777 & $0.016^{*}$ & $0.732(0.568-0.944)$ \\
CVFT & -0.036 & 0.057 & 0.392 & 0.531 & $0.965(0.864-1.079)$ \\
DR & -0.338 & 0.143 & 5.588 & $0.018^{*}$ & $0.713(0.538-0.944)$ \\
ADAS-Cog & 0.042 & 0.067 & 0.388 & 0.533 & $1.043(0.914-1.189)$ \\
CSDD & 0.152 & 0.110 & 1.895 & 0.169 & $1.164(0.938-1.445)$ \\
CIRS & 0.092 & 0.142 & 0.421 & 0.517 & $1.096(0.831-1.447)$ \\
Constant & 4.118 & 5.799 & 0.504 & 0.478 & 61.419 \\
\hline
\end{tabular}

Note. Good model fit was evidenced by non-statistically significant results on the Hosmer-Lemeshow test, $\chi^{2}=6.012, \mathrm{df}=8, \mathrm{p}=0.646 ;$ Cox and Snell $R^{2}=0.161 ;$ Nagelkerke $R^{2}=0.400$. $* \mathrm{p}=\leq 0.05$

$\mathrm{Edu}$ yrs = years of education; $\mathrm{CMMSE}=$ Cantonese version of the Mini-mental State Examination; $\mathrm{CVFT}=$ Category Verbal Fluency Test; DR=Delayed Recall; ADAS-Cog $=$ Chinese version of the Alzheimer's Disease Assessment Scale-Cognitive Subscale; CSDD = Cornell Scale for Depression in Dementia; CIRS $=$ Cumulative Illness Rating Scale; $\mathrm{CI}=$ confidence interval .

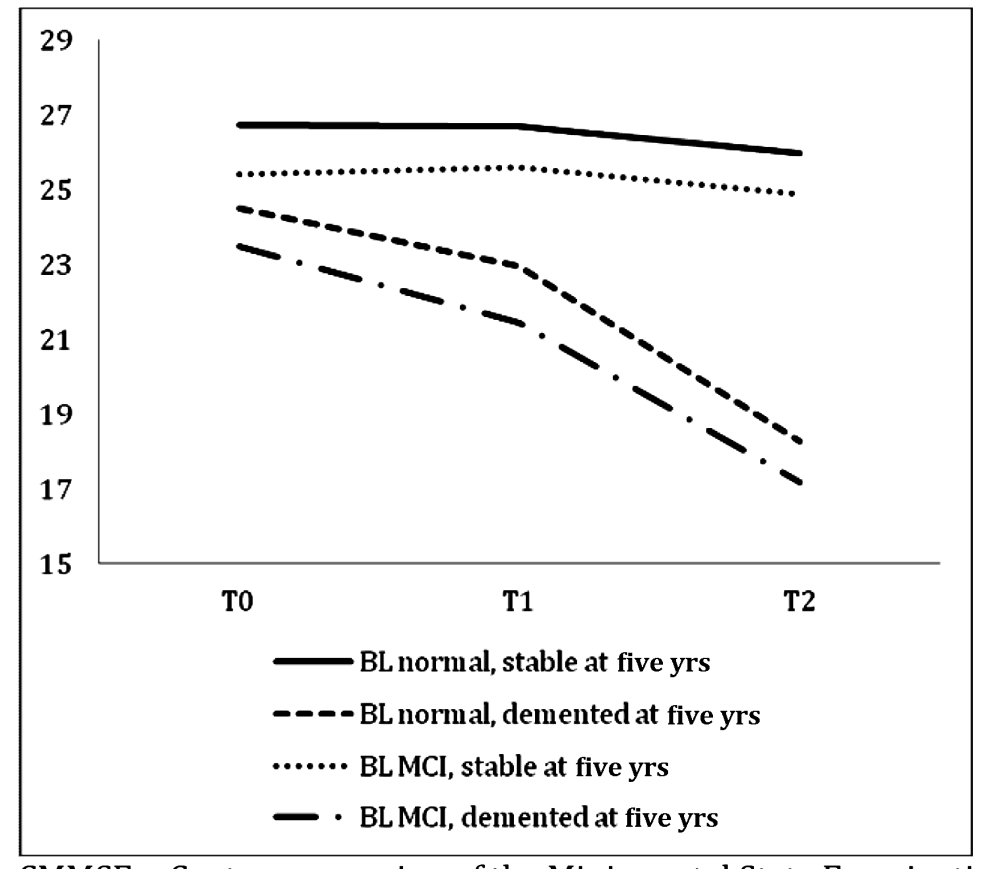

CMMSE $=$ Cantonese version of the Mini-mental State Examination

Figure 2. Trajectories of the CMMSE score over five years. 
predictors of subsequent incident dementia at five years.

\section{Decline in cognitive profile in two years predicting progression over five years}

The sensitivity and specificity were $72.3 \%-77.2 \%$ if we evaluate a 1-SD drop in either CMMSE or CVFT alone to predict dementia at five years. A significant drop in either CMMSE or CVFT or both tests predicts dementia at five years with a sensitivity of $91.5 \%$. A stable MMSE and CVFT predicted a $91 \%$ chance of maintaining stable cognitive state at five years (Table 5).

The analyses were repeated separating the participants into baseline normal and MCI groups. In the baseline normal group, a significant drop in both CMMSE and CVFT predicts dementia at five years with a sensitivity of $84.6 \%$ and a specificity of $88.0 \%$. In the baseline MCI group, a significant drop in both CMMSE and CVFT predicts dementia at five years with a sensitivity of $88.9 \%$ and a specificity of $83.3 \%$.

\section{Discussion}

A higher proportion of baseline MCI participants $(31.5 \%)$ than baseline normal participants $(7.9 \%)$ progressed to dementia after five years. The estimates of annual conversion rates of $1.6 \%$ and $6.3 \%$ for normal and MCI groups are comparable with previous studies. Our findings might be slightly conservative as participants who were not available or declined follow-up had lower cognitive profiles at baseline, suggesting that they had higher risks developing dementia. In fact, our results were consistent with conversion rates reported in other population-based studies, which reported annual conversion from MCI to dementia to be $3 \%-16 \%$ (Ritchie et al., 2001; Amieva et al., 2004; Busse et al., 2006). Petersen (2003) reports that after approximately six years, $80 \%$ of the MCI cohort has progressed to dementia, but the conversion figure was based on a clinical sample, which might be more impaired than population-based samples.

For participants who developed dementia at five year, the drop in CMMSE in the first two years was significant, and the mean drop was around 2 points. Only few population-based studies explicitly examined the predictive accuracy of a decline in total MMSE score over time to future development of dementia. In a study in Gothenburg, a decline of 4 points over three years was found to be the best cut-off for dementia in participants with age 85-88, and the participants who progressed to dementia had an average annual MMSE drop of 2.3 points (Acvarsson and Skoog, 2000). In the AMSTEL project, $-3 /-4$ points had the highest sensitivity, while $-5 /-6$ points had the highest specificity over one year (Schmand et al., 1995). In another study in Leipzig, the best cut-off drop in raw MMSE score was -1 point over two years to predict dementia at nine years (Hensel et al., 2009). The findings suggested that global cognitive function as assessed by MMSE could be a cognitive predictor for decline in a community sample.

The decline in the CVFT score was also significant in participants who progressed to dementia. Interestingly, the decline in CVFT was present in the first two years over the five-year period. Detecting a significant decline in CVFT may indicate risk of developing dementia in the next few years. CVFT involves semantic memory and executive function. In our study, we were not able to tell whether memory declined before T0, but it was clear that a significant decline in CVFT scores to a low level comparable to over $1 \mathrm{SD}$ below cognitive normal participants in two years predicted subsequent progression to dementia. Previous studies also reported that cognitive decline would start as early as seven years before clinical diagnosis of dementia (Grober et al., 2008). We also echoed the findings that cognitive decline was present many years before clinical diagnosis of dementia. Also, the trajectories of decline of specific cognitive domains are different and may have an impact on the predictability of disease progression. The sensitivity to changes with the dementing process varies among different cognitive tests, which may explain the different trajectories observed in CMMSE versus CVFT performance. Delayed recall has been found to be a strong cognitive predictor of future decline. However, the decline in this test had reached a floor effect early in the cognitive decline process in participants of this study.

Our results offered useful information on cognitive predictors for decline in a community sample. CMMSE and CVFT were chosen for analysis in combination because they are convenient to administer in clinical practice and the completion rate of these tests was high. For communitydwelling participants assessed serially, a drop in MMSE scores of 2 points and CVFT of 6 points (for baseline normal participants) and 4 points (for baseline MCI participants) in two years would be predictive of subsequent progression to dementia in the next few years. If a 1-SD drop in either test predicted subsequent dementia with a sensitivity of $72.3 \%-76.6 \%$ only; but when both tests were considered together, a 1-SD drop in either test in two years predicted $91.5 \%$ dementia cases at five years. A stable performance in either test predicted dementia-free at five years with a specificity of 
Table 5. CMMSE and CVFT drop at two years and progression to dementia at five years (all participants)

\begin{tabular}{|c|c|c|c|}
\hline$\geq 1 \mathrm{SD}$ DROP & $\begin{array}{l}\text { HAD } \\
\text { DEMENTIA AT } \\
\text { FIVE YEARS }\end{array}$ & $\begin{array}{l}\text { WITHOUT } \\
\text { DEMENTIA AT } \\
\text { FIVE YEARS }\end{array}$ & \\
\hline \multicolumn{4}{|l|}{ MMSE } \\
\hline Yes & 36 & 74 & $\mathrm{PPV}=32.7 \%$ \\
\hline No & $\begin{array}{l}11 \\
\text { Sens }=76.6 \%\end{array}$ & $\begin{array}{l}250 \\
\text { Spec }=77.2 \%\end{array}$ & $\mathrm{NPV}=95.8 \%$ \\
\hline \multicolumn{4}{|l|}{ CVFT } \\
\hline Yes & 34 & 81 & $P P V=29.6 \%$ \\
\hline No & $\begin{array}{l}13 \\
\text { Sens }=72.3 \%\end{array}$ & $\begin{array}{l}255 \\
\text { Spec }=75.9 \%\end{array}$ & $\mathrm{NPV}=95.1 \%$ \\
\hline \multicolumn{4}{|c|}{ CMMSE and CVFT } \\
\hline Yes & 27 & 29 & $\mathrm{PPV}=48.2 \%$ \\
\hline No & $\begin{array}{l}20 \\
\text { Sens }=57.4 \%\end{array}$ & $\begin{array}{l}295 \\
\text { Spec }=91.0 \%\end{array}$ & $\mathrm{NPV}=93.7 \%$ \\
\hline \multicolumn{4}{|c|}{ CMMSE and/or CVFT } \\
\hline Yes & 43 & 123 & $P P V=25.9 \%$ \\
\hline No & $\begin{array}{l}4 \\
\text { Sens }=91.5 \%\end{array}$ & $\begin{array}{l}201 \\
\text { Spec }=62.0 \%\end{array}$ & $\mathrm{NPV}=98.0 \%$ \\
\hline
\end{tabular}

$\mathrm{CMMSE}=$ Cantonese version of the Mini-mental State Examination; CVFT $=$ Category Verbal Fluency Test; $\mathrm{SD}=$ standard deviation; Sens = sensitivity; $\mathrm{Spec}=$ specificity; $\mathrm{PPV}=$ positive predictive value; NPV = negative predictive value.

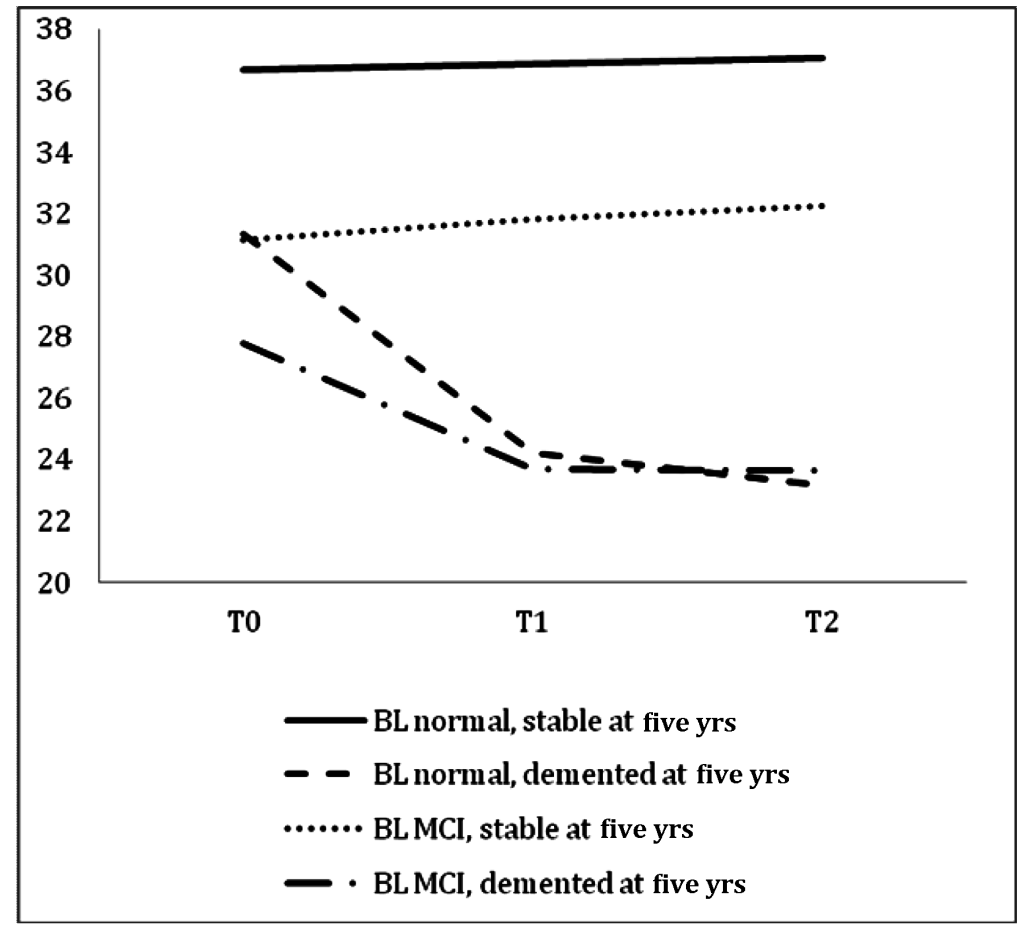

CVFT = Category Verbal Fluency Test.

Figure 3. Trajectories of the CVFT score over five years.

$75.9 \%-77.2 \%$ only, but a stable performance in both tests at two years predict a $91 \%$ chance of not progressing to dementia at five years. When analyzing the baseline normal and MCI participants separately, the predictive power of the early decline in both tests became more significant than considering either test alone. The two tests are simple to administer. This relatively simple algorithm may serve as a screening tool in the community for early identification of individuals at risk of developing dementia, and help in the selection of potential participants for interventions 
for dementia prevention. This study also provided additional information when compared with a local study (Lam et al., 2008c). The current study offered a longer follow-up for a population-based random sample, while the previous study provided data on volunteers with subjective memory complaints.

There are a number of limitations concerning the study design. The follow-up interval between the two follow-up assessments was not uniform. A more reliable trajectory of cognitive changes over time could only be deduced if data are available for more follow-up time points with regular followup interval. Participants lost to follow-up were older and had poorer cognitive performance at baseline. The conversion rate to dementia was likely an underestimation of the actual figure in the population. Moreover, the diagnosis of dementia was not ascertained with neuroimaging investigation. The profile of cognitive decline would be different for people with dementia of Alzheimer's type and vascular dementia. However, it would be difficult to delineate a group with "pure" Alzheimer's in this locality, because the local prevalence of cardiovascular events is high and the mixed-type dementia is common.

This study adds further data to the existing literature on the trajectory of cognitive profile in older adults, both in cases with normal aging and those who progressed to dementia eventually. It helps giving insight and evidence to how the screening and timely intervention can be modeled for the best effect.

\section{Conflict of interest}

None.

\section{Description of authors' roles}

Candy H. Y. Wong formulated the research question, designed the study, collected the data, was responsible for the statistical design of the study, carried out the statistical analysis, and wrote the paper. Grace T. Y. Leung designed the study and collected the data. Ada W. T. Fung and W. C. Chan collected the data. Linda C. W. Lam formulated the research question, designed the study, supervised the data collection, and wrote the paper.

\section{References}

Acvarsson, O. and Skoog, I. (2000). A longitudinal population study of the mini-mental state examination in the very old: relation to dementia and education. Dementia and Geriatric Cognitive Disorders, 11, 166-175.
Alexopoulos, G. S., Abrams, R. C., Young, R. C. and Shamoian, C. A. (1988). Cornell Scale for Depression in Dementia. Biological Psychiatry, 23, 271284.

American Psychiatric Association. (2000). Diagnostic and Statistical Manual of Mental Disorders, 4rth edn, Text Revision (DSM-IV-TR). Washington, DC: American Psychiatric Association.

Amieva, H. et al. (2004). Annual rate and predictors of conversion to dementia in participants presenting mild cognitive impairment criteria defined according to a population-based study. Dementia and Geriatric Cognitive Disorders, 18, 87-93.

Bowie, C. R. and Harvey, P. D. (2006). Administration and interpretation of the trail making test. Nature Protocols, 1, 2277-2281.

Busse, A., Angermeyer, M. C. and Riedel-Heller, S. G. (2006). Progression of mild cognitive impairment to dementia: a challenge to current thinking. The British fournal of Psychiatry, 189, 399-404.

Chen, P., Ratcliff, G., Belle, S. H., Cauley, J. A., DeKosky, S. T. and Ganguli, M. (2001). Cognitive tests that best discriminate between presymptomatic $\mathrm{AD}$ and those who remain nondemented. Neurology, 55, 1847-1853.

Chiu, H. F. K., Lee, H. C. B., Chung, D. and Kwong, P. K. (1994). Reliability and validity of the Cantonese version of the Mini-Mental State Examination: a preliminary study. Fournal of Hong Kong College of Psychiatrists, 4 (Suppl. 2), 25-28.

Chiu, H. F. K. et al. (1997). The modified fuld verbal fluency test: a validation study in Hong Kong. Fournals of Gerontology, Series B, Psychological Sciences and Social Sciences, 52B, 247-250.

Chu, L. W., Chiu, K. C., Hui, S. L., Yu, G. K., Tsui, W. J. and Lee, P. W. (2000). The reliability and validity of the Alzheimer's Disease Assessment Scale Cognitive Subscale (ADAS-Cog) among the elderly Chinese in Hong Kong. Annals of the Academy of Medicine, Singapore, 29, 474-485.

Conwell, Y., Forbes, N. T., Cox, C. and Caine, E. D. (1993). Validation of a measure of physical illness burden at autopsy: the Cumulative Illness Rating Scale. Fournal of American Geriatric Society, 41, 38-41.

Ebly, E., Hogan, D. and Parhad, I. (1995). Cognitive impairment in the non-demented elderly: results from the Canadian study of health and aging. Archives of Neurology, $52,612-619$.

Folstein, M. F., Folstein, S. E. and McHugh, P. R. (1975). "Mini-mental state". A practical method for grading the cognitive state of patients for the clinician. Fournal of Psychiatric Research, 12, 189-198.

Galvin, J. E. et al. (2005). Predictors of preclinical Alzheimer's disease and dementia: a clinicopathologic study. Archives of Neurology, 62, 758-765.

Grober, E., Hall, C. B., Lipton, R. B., Zonderman, A. B., Resnick, S. M. and Kawas, C. (2008). Memory impairment, executive dysfunction, and intellectual decline in preclinical Alzheimer's disease. Fournal of International Neuropsychological Society, 14, 266-278.

Hall, C. B., Lipton, R. B., Sliwinski, M. and Stewart, W. F. (2000). A change point model for estimating the 
onset of cognitive decline in preclinical Alzheimer's disease. Statistics in Medicine, 19, 1555-1566.

Hensel, A., Luck, T., Luppa, M., Glaesmer, H., Angermeyer, M. C. and Riedel-Heller, S. G. (2009). Does a reliable decline in Mini Mental State Examination total score predict dementia? Diagnostic accuracy of two reliable change indices. Dementia and Geriatric Cognitive Disorders, 27, 50-58.

Howieson, D. B., Dame, A., Camicioli, R., Sexton, G., Payami, H. and Kaye, J. A. (1997). Cognitive markers preceding Alzheimer's dementia in the healthy oldest old. Fournal of American Geriatrics Society, 45, 584-589.

Johnson, D. K., Storandt, M., Morris, J. C. and Gavin, J. E. (2009). Longitudinal study of the transition from healthy aging to Alzheimer disease. Archives of Neurology, 66, 1254-1259.

Lam, L. C. W. et al. (2008a). Prevalence of very mild and mild dementia in community-dwelling older Chinese people in Hong Kong. International Psychogeriatrics, 20, 135-148.

Lam, L. C. W. et al. (2008b). Use of clinical dementia rating in detecting early cognitive deficits in a community-based sample of Chinese older persons in Hong Kong. Alzheimer Disease and Associated Disorders, 22, 153-157.

Lam, L. C. W. et al. (2008c). Screening of mild cognitive impairment in Chinese older adults - a multistage validation of the Chinese abbreviated mild cognitive impairment test. Neuroepidemiology, 30, 6-12.

Lam, L. C. W. et al. (2010). Combined clinical and cognitive criteria to identify mild cognitive impairment in a Southern
Chinese Community. Alzheimer Disease and Associated Disorders, 24, 343-347.

Leung, G. T. Y. (2008). Examining the association between participation in late-life leisure activities and cognitive function in community-swelling elderly Chinese in Hong Kong. Research dissertation, the Hong Kong College of Psychiatrists.

Petersen, R. C. (2003). Mild Cognitive Impairment. Oxford: Oxford University Press.

Ritchie, K., Artero, S. and Touchon, J. (2001). Classification criteria for mild cognitive impairment. A population-based validation study. Neurology, 56, 37-42.

Rosen, W. G., Mohs, R. C. and Davis, K. L. (1984). A new rating scale for Alzheimer's disease. American fournal of Psychiatry, 141, 1356-1364.

Rubin, E. H., Storandt, M., Miller, J. P., Kinscherf, D. A., Grant, E. A. C. and Berg, L. (1998). A Prospective study of cognitive function and onset of dementia in cognitively healthy elders. Archives of Neurology, 55, 395-401.

Schmand, B., Lindeboom, J., Launer, L., Dinkgreve, M., Hooijer, C. and Jonker, C. (1995). What is a significant score change on the Mini-Mental State examination? International Fournal of Geriatric Psychiatry, 10, 411-414.

Small, B. J., Fratiglioni, L., von Strauss, E. and Bäckman, L. (2003). Terminal decline and cognitive performance in very old age: does cause of death matter? Psychology and Aging, 18, 193-202.

Wilson, R. S., Beckett, L. A., Bienias, J. L., Evans, D. A. and Bennett, D. A. (2003). Terminal decline in cognitive function. Neurology, 60, 1782-1787. 